\title{
Estimation of the exponential distribution based on multiply Type I hybrid censored sample ${ }^{\dagger}$
}

\author{
Kyeongjun Lee ${ }^{1} \cdot$ Hokeun $\mathrm{Sun}^{2} \cdot$ Youngseuk $\mathrm{Cho}^{3}$ \\ ${ }^{123}$ Department of Statistics, Pusan National University \\ Received 13 March 2014, revised 7 April 2014, accepted 12 April 2014
}

\begin{abstract}
The exponential distibution is one of the most popular distributions in analyzing the lifetime data. In this paper, we propose multiply Type I hybrid censoring. And this paper presents the statistical inference on the scale parameter for the exponential distribution when samples are multiply Type I hybrid censoring. The scale parameter is estimated by approximate maximum likelihood estimation methods using two different Taylor series expansion types (AMLE,$A_{I}$ AMLE II $)$. We also obtain the maximum likelihood estimator (MLE) of the scale parameter $\sigma$ under the proposed multiply Type I hybrid censored samples. We compare the estimators in the sense of the root mean square error (RMSE). The simulation procedure is repeated 10,000 times for the sample size $n=20$ and 40 and various censored schemes. The AMLE $\mathrm{II}_{\mathrm{II}}$ is better than AMLE $\mathrm{I}_{\mathrm{I}}$ in the sense of the RMSE.
\end{abstract}

Keywords: Approximate maximum likelihood estimator, exponential distribution, multiply Type I hybrid censored sample, Type I hybrid censored sample.

\section{Introduction and notation}

Consider an exponential distribution with probability density function (p.d.f.)

$$
g_{X}(x ; \sigma)=\frac{1}{\sigma} e^{-x / \sigma}, \quad x \geq 0, \quad \sigma>0,
$$

and cumulative distribution function (c.d.f.)

$$
G_{X}(x ; \sigma)=1-e^{-x / \sigma}, \quad x \geq 0, \quad \sigma>0 .
$$

The estimation of the parameters of the exponential distribution based on censored samples has been investigated by many authors such as Balakrishnan and Sandu (1996), Kang and Cho (1998), Kang and Park (2005), Singh and Kumar (2007), Asgharzadeh (2009), Shin et al. (2010), and Lee et al. (2012). Balakrishnan and Sandu (1996) derived the best linear

$\dagger$ This work was supported for two years by Pusan National University Research Grant.

1 Instructor, Department of Statistics, Pusan National University, Busan 609-735, Korea.

2 Assistant professor, Department of Statistics, Pusan National University, Busan 609-735, Korea.

3 Corresponding author: Professor, Department of Statistics, Pusan National University, Busan 609-735, Korea. E-mail: choys@pusan.ac.kr 
unbiased estimators (BLUEs) and maximum likelihood estimation under general progressive Type II censored sample. Kang and Cho (1998) obtained the MRE for exponential distribution under general progressive Type II censored samples. Kang and Park (2005) derived an approximate maximum likelihood estimator (AMLE) for the exponentiated exponential distribution based on multiply Type II censored samples. Singh and Kumar (2007) derived a Bayes estimation based on multiply Type II censoring scheme. Asgharzadeh (2009) derived an AMLE for the scaled generalized exponential distribution under progressive Type II censoring. Shin et al. (2010) considered the parameter estimation for exponential distribution under progressive Type I interval censoring. Lee et al. (2012) considered the parameter estimation for exponential distribution under Multiply progressive Type II censoring.

There are many situation in life testing experiments in which units are lost or removed from experimentation before failure. The loss may occur carelessly or unconsciously. For example, carelessly loss may occur in the case of accidental breakage of an experimental unit. Epstein (1954) introduced a Type I hybrid censoring scheme in which the test is terminated at a random time $T^{*}=\min \left\{X_{r: n}, T\right\}$, where $X_{r: n}$ denotes the $r$-th ordered failure time when the sample size is $n, r \in\{1,2, \cdots, n\}$ and $T \in(0, \infty)$ are pre-fixed. A schematic representation of the Type I hybrid censoring scheme is presented in Figure 1.1.

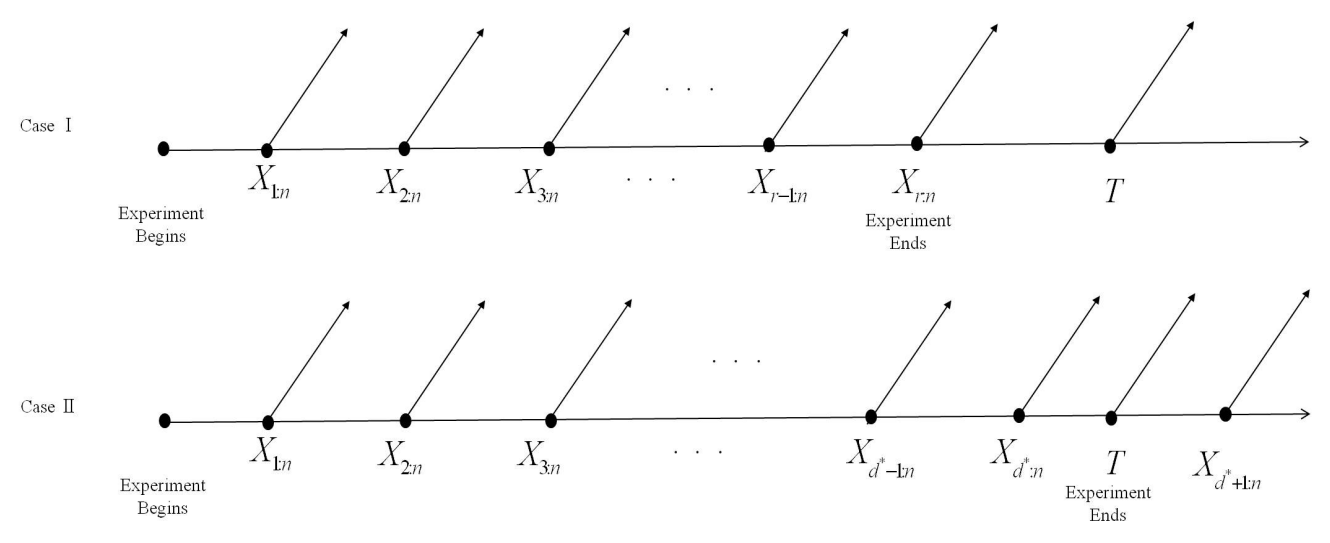

Figure 1.1 The Type I hybrid censoring schemes

Under Type I censored sample, however, some units can be failed between two points of observation with exact times of failure of these units unobserved. For example, loss may arise in life-testing experiments when the failure times of some units were not observed due to mechanical or experimental difficulties. Therefore, the new censoring method is needed.

This study has two aims. The first is to propose a multiply Type I hybrid censoring scheme. The second aim is to consider the MLE of the scale parameter $\sigma$ when the data are multiply Type I hybrid censored samples. However, MLE cannot be obtained in a closed form. We use the AMLEs as an approximate estimator of scale parameter. The rest of the paper is organized as follows. In section 2, we propose the multiply Type I hybrid censoring scheme. In section 3, we derive some AMLEs of the $\sigma$ for the exponential distribution under the proposed multiply Type I hybrid censored samples. The $\sigma$ is estimated by approximation method using two different Taylor series expansion types. 


\section{Multiply Type I hybrid censoring}

Under the Type I hybrid censoring scheme, suppose the experimenter fails to observe the middle $l$ observations. Now, let

$$
\begin{array}{cc}
\text { Case } & \text { I }:\left\{X_{a_{1}: n}<X_{a_{2}: n}<\cdots<X_{a_{s-1}: n}<X_{a_{s}: n}<T\right\} \text { if } X_{a_{s}: n}<T \\
\text { Case } & \text { II }:\left\{X_{a_{1}: n}<X_{a_{2}: n}<\cdots<X_{a_{d-1}: n}<X_{a_{d}: n}<T<X_{a_{d}+1: n}<\cdots<X_{a_{s}: n}\right\} \\
& \quad \text { if } d<s, \text { and } X_{a_{d}: n}<T<X_{a_{d}+1: n .}
\end{array}
$$

Note that, $X_{a_{i}: n}$ denote the $a_{i}$ th failure. $a_{s}=r+\sum_{i=1}^{s-1} l_{i}$ if $r+\sum_{i=1}^{s-1} l_{i}<n$, and $a_{s}=n$ if $r+\sum_{i=1}^{s-1} l_{i} \geq n . l_{i}=a_{i+1}-a_{i}+1$, where $i=1,2, \cdots, s-1$, and $l=\sum_{i=1}^{s-1} l_{i}$. In case II, $X_{a_{d}: n}<T<X_{a_{d+1: n}}$ means that the $a_{d}$ th failure took place before $T$, and no failure took place between $X_{a_{d}: n}$ and $T$. A schematic representation of the multiply Type I hybrid censoring scheme is presented in Figure 2.2.

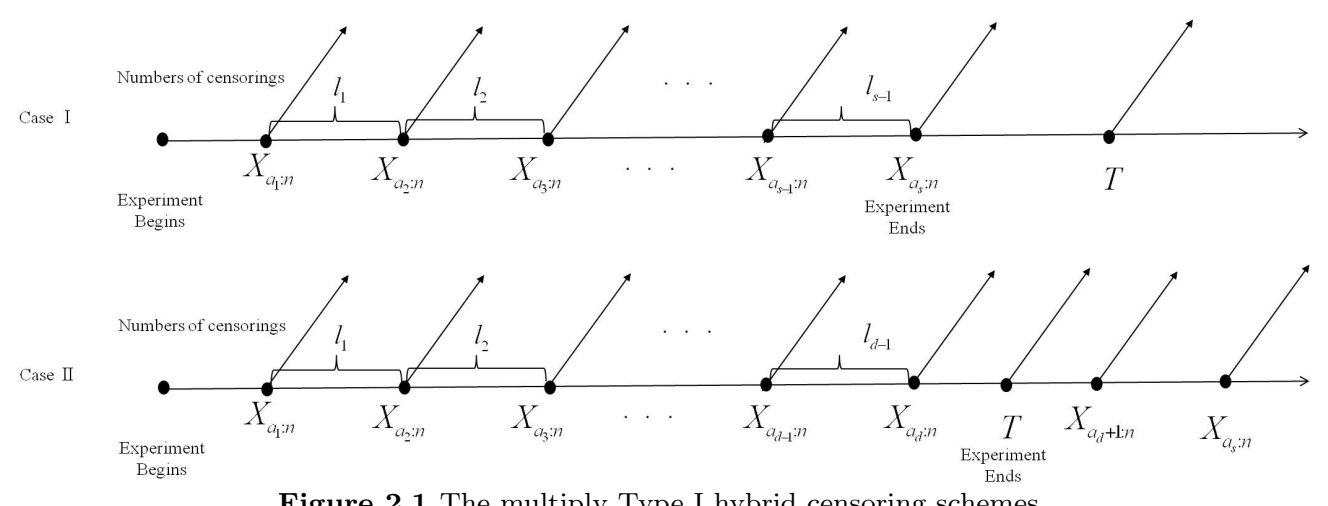

Figure 2.1 The multiply Type I hybrid censoring schemes

Let $a_{1}=1$, the likelihood function based on (2.1) and (2.2) is given by

Case I

$$
L \propto \prod_{i=1}^{s} g\left(x_{a_{i}: n}\right)\left[1-G\left(x_{a_{i}: n}\right)\right]^{n-a_{s}} \prod_{i=2}^{s}\left[G\left(x_{a_{i}: n}\right)-G\left(x_{a_{i-1}: n}\right)\right]^{l_{i-1}}
$$

Case II

$$
L \propto \prod_{i=1}^{d} g\left(x_{a_{i}: n}\right)[1-G(T)]^{n-a_{d}} \prod_{i=2}^{d}\left[G\left(x_{a_{i}: n}\right)-G\left(x_{a_{i-1}: n}\right)\right]^{l_{i-1}} .
$$

Therefore, case I and case II can be combined, and can be written as

$$
L \propto \prod_{i=1}^{u} g\left(x_{a_{i}: n}\right)[1-G(D)]^{n-a_{u}} \prod_{i=2}^{u}\left[G\left(x_{a_{i}: n}\right)-G\left(x_{a_{i-1}: n}\right)\right]^{l_{i-1}}
$$

where $u$ denotes the number of failures; and $D=x_{a_{s}: n}$ if $x_{a_{s}: n}<T$, and $D=T$ if $x_{a_{s}: n}>T$.

Let $Z_{a_{i}: n}=X_{a_{i}: n} / \sigma$ and then the variables $Z_{a_{i}: n}$ have a standard exponential distribution with pdf $f\left(z_{a_{i}: n}\right)=e^{-z_{a_{i}: n}}, z_{a_{i}: n} \geq 0$, and $\operatorname{cdf} F\left(z_{a_{i}: n}\right)=1-e^{-z_{a_{i}: n}}, z_{a_{i}: n} \geq 0$, we may rewrite the above likelihood function as

$$
L \propto \sigma^{-u} \prod_{i=1}^{u} f\left(z_{a_{i}: n}\right)[1-F(D)]^{n-a_{u}} \prod_{i=2}^{u}\left[F\left(z_{a_{i}: n}\right)-F\left(z_{a_{i-1}: n}\right)\right]^{l_{i-1}} .
$$


Upon substituting for the functions $F(\cdot)$ and $f(\cdot)$, we obtain from $(2.4)$ that

$$
\begin{aligned}
\ln L \propto & -u \ln \sigma+\sum_{i=1}^{u}\left\{\ln f\left(z_{a_{i}: n}\right)+\left(n-a_{u}\right) \ln [1-F(D)]\right\} \\
& +\sum_{i=2}^{u} l_{i-1} \ln \left[F\left(z_{a_{i}: n}\right)-F\left(z_{a_{i-1}: n}\right)\right] .
\end{aligned}
$$

On differentiating the log-likelihood functions with respect to $\sigma$ of (2.5) and equation to zero, we obtain the estimating equations as

$$
\begin{aligned}
\frac{\partial \ln L}{\partial \sigma}= & -\frac{1}{\sigma}\left[u-\sum_{i=1}^{u} z_{a_{i}: n}-\left(n-a_{u}\right) z_{D}\right. \\
& \left.+\sum_{i=2}^{u} l_{i-1}\left\{\frac{f\left(z_{a_{i}: n}\right) z_{a_{i}: n}-f\left(z_{a_{i-1}: n}\right) z_{a_{i-1}: n}}{F\left(z_{a_{i}: n}\right)-F\left(z_{a_{i-1}: n}\right)}\right\}\right] \\
= & 0 .
\end{aligned}
$$

We can find the MLE $\hat{\sigma}$ of $\sigma$ that maximize the log-likelihood function in (2.5) by solving (2.6). Since (2.6) cannot be solved explicitly, some numerical methods must be employed. So we use the Newton-Raphson method to obtain the numerical solution of (2.6).

\section{Approximate maximum likelihood estimators}

Since (2.6) cannot be solved explicitly, it will be desirable to consider an approximation to the likelihood equations which provide us with explicit estimators for $\sigma$. See for example the work of Cho et al. (2013), Kang and Lee (2013), and Lee and Lee (2012).

Let $\xi_{a_{i}: n}=F^{-1}\left(p_{a_{i}: n}\right)=-\ln \left(1-p_{a_{i}: n}\right)=-\ln \left(q_{a_{i}: n}\right)$, where

$$
p_{a_{i}: n}=\frac{a_{i}}{n+1}, i=1, \cdots, s .
$$

First, we expand the functions $\frac{f\left(z_{a_{i}: n}\right)}{\left\{F\left(z_{a_{i}: n}\right)-F\left(z_{a_{i-1}: n}\right)\right\}}$ and $\frac{f\left(z_{a_{i-1}: n}\right)}{\left\{F\left(z_{a_{i}: n}\right)-F\left(z_{a_{i-1}: n}\right)\right\}}$ in Taylor series around the points $\xi_{a_{i}: n}$.

Then, we can approxmate the functions by

$$
\begin{aligned}
& \frac{f\left(z_{a_{i}: n}\right)}{F\left(z_{a_{i}: n}\right)-F\left(z_{a_{i-1}: n}\right)} \simeq \alpha_{1 i}+\beta_{1 i} z_{a_{i}: n}+\gamma_{1 i} z_{a_{i-1}: n}, \\
& \frac{f\left(z_{a_{i-1}: n}\right)}{F\left(z_{a_{i}: n}\right)-F\left(z_{a_{i-1}: n}\right)} \simeq \alpha_{2 i}+\beta_{2 i} z_{a_{i}: n}+\gamma_{2 i} z_{a_{i-1}: n},
\end{aligned}
$$

where

$$
\begin{aligned}
\alpha_{1 i} & =\frac{q_{a_{i}: n}}{p_{a_{i}: n}-p_{a_{i-1}: n}}\left[1+\xi_{a_{i}: n}+\frac{q_{a_{i}: n} \xi_{a_{i}: n}-q_{a_{i-1}: n} \xi_{a_{i-1}: n}}{p_{a_{i}: n}-p_{a_{i-1}: n}}\right], \\
\beta_{1 i} & =-\frac{q_{a_{i}: n}}{p_{a_{i}: n}-p_{a_{i-1}: n}}\left[1+\frac{q_{a_{i}: n}}{p_{a_{i}: n}-p_{a_{i-1}: n}}\right], \\
\gamma_{1 i} & =\frac{q_{a_{i}: n} q_{a_{i-1}: n}}{\left(p_{a_{i}: n}-p_{a_{i-1}: n}\right)^{2}}
\end{aligned}
$$




$$
\begin{aligned}
\alpha_{2 i} & =\frac{q_{a_{i-1}: n}}{p_{a_{i}: n}-p_{a_{i-1}: n}}\left[1+\xi_{a_{i-1}: n}+\frac{q_{a_{i}: n} \xi_{a_{i}: n}-q_{a_{i-1}: n} \xi_{a_{i-1}: n}}{p_{a_{i}: n}-p_{a_{i-1}: n}}\right], \\
\beta_{2 i} & =-\frac{q_{a_{i}: n} q_{a_{i-1}: n}}{\left(p_{a_{i}: n}-p_{a_{i-1}: n}\right)^{2}}, \\
\gamma_{2 i} & =-\frac{q_{a_{i-1}: n}}{p_{a_{i}: n}-p_{a_{i-1}: n}}\left[1-\frac{q_{a_{i-1}: n}}{p_{a_{i}: n}-p_{a_{i-1}: n}}\right] .
\end{aligned}
$$

By substituting the equations (3.1) and (3.2) into (2.6), we may approximate the equation in $(2.6)$ by

$$
\begin{aligned}
\frac{\partial \ln L}{\partial \sigma} \simeq & \frac{\partial \ln L^{*}}{\partial \sigma}=-\frac{1}{\sigma}\left[u-\sum_{i=1}^{u} z_{a_{i: n}}-\left(n-a_{u}\right) z_{D}\right. \\
& +\sum_{i=2}^{u} l_{i-1}\left\{\left(\alpha_{1 i}+\beta_{1 i} z_{a_{i: n}}+\gamma_{1 i} z_{a_{i-1: n}}\right) z_{a_{i: n}}\right. \\
& \left.\left.-\left(\alpha_{2 i}+\beta_{2 i} z_{a_{i: n}}+\gamma_{2 i} z_{a_{i-1: n}}\right) z_{a_{i-1: n}}\right\}\right] \\
& =0
\end{aligned}
$$

(3.3) is a quadratic equation in $\sigma$, with its roots given by

$$
\hat{\sigma}_{\mathrm{I}}=\frac{B_{\mathrm{I}}+\sqrt{B_{\mathrm{I}}^{2}-4 u C_{\mathrm{I}}}}{2 u},
$$

where

$$
\begin{aligned}
& B_{\mathrm{I}}=\sum_{i=1}^{u} x_{a_{i}: n}+\left(n-a_{u}\right) D-\sum_{i=2}^{u} l_{i-1}\left(\alpha_{1 i} x_{a_{i}: n}-\alpha_{2 i} x_{a_{i-1: n}}\right), \\
& C_{\mathrm{I}}=\sum_{i=2}^{u} l_{i-1}\left(\beta_{1 i} x_{a_{i}: n}^{2}+2 \gamma_{1 i} x_{a_{i}: m} x_{a_{i-1: n}}-\gamma_{2 i} x_{a_{i-1: n}}^{2}\right) .
\end{aligned}
$$

Since $\beta_{1 i} x_{a_{i}: n}^{2}+2 \gamma_{1 i} x_{a_{i}: n} x_{a_{i-1: n}}-\gamma_{2 i} x_{a_{i-1: n}}^{2} \leq 0$, the estimator $\hat{\sigma}_{\mathrm{I}}$ is always positive.

To develop interval estimation for the $\sigma$, we need to compute the asymptotic variance which is related to the Fisher information. From (3.3), we obtain

$$
E\left(-\frac{\partial^{2} \ln L^{*}}{\partial \sigma^{2}}\right)=\frac{D^{*}}{\sigma^{2}}
$$

where

$$
\begin{aligned}
D^{*}= & 2\left[\sum_{i=1}^{u} E\left(z_{a_{i}: n}\right)+\left(n-a_{u}\right) E\left(z_{D}\right)-\sum_{i=2}^{u} l_{i-1}\left(\alpha_{1 i} E\left(z_{a_{i}: n}\right)-\alpha_{2 i} E\left(z_{a_{i-1}: n}\right)\right)\right] \\
& -3 \sum_{i=2}^{u} l_{i-1}\left(\beta_{1 i} z_{a_{i}: n}^{2}+2 \gamma_{1 i} z_{a_{i}: n} z_{a_{i-1}: n}-\gamma_{2 i} z_{a_{i-1}: n}^{2}\right)-u .
\end{aligned}
$$


This quantity may be computed by using Balakrishnan and Cohen (1991):

$$
\begin{aligned}
& E\left(z_{i: n}\right)=\frac{n !}{(i-1) !(n-i) !} \sum_{m=0}^{i-1}(-1)^{m}\left(\begin{array}{c}
i-1 \\
m
\end{array}\right)(n+m-i+1)^{-2}, \\
& E\left(z_{i: n}^{2}\right)=2 \frac{n !}{(i-1) !(n-i) !} \sum_{m=0}^{i-1}(-1)^{m}\left(\begin{array}{c}
i-1 \\
m
\end{array}\right)(n+m-i+1)^{-3}, \\
& E\left(z_{i: n} z_{j: n}\right)=K \sum_{m=0}^{i-1} \sum_{v=0}^{j-i-1}(-1)^{j-i-1-v+m}\left(\begin{array}{c}
i-1 \\
m
\end{array}\right)\left(\begin{array}{c}
j-i-1 \\
v
\end{array}\right) \phi(a, b),
\end{aligned}
$$

where $K=n ! /(i-1) !(j-i-1) !(n-j) !, \phi(a, b)=(a b)^{-2} \operatorname{IB}_{a /(a+b)}(2,2), a=m+v+1$, $b=n-i-v, 1 \leq i<j \leq n, b \leq a$, and $\operatorname{IB}_{p}\left(d_{1}, d_{2}\right)$ is incomplete beta function.

The asymptotic variance of the AMLE $\mathrm{I}$ of $\sigma$ is given by

$$
\operatorname{Var}\left(\hat{\sigma}_{\mathrm{I}}\right) \simeq \frac{1}{E\left[-\partial^{2} \ln L^{*} / \partial \sigma^{2}\right]}
$$

Second, we expand the functions $\frac{f\left(z_{a_{i}: n}\right) z_{a_{i}: n}-f\left(z_{a_{i-1}: n}\right) z_{a_{i-1}: n}}{F\left(z_{a_{i}: n}\right)-F\left(z_{a_{i-1}: n}\right)}$ in Taylor series around the points $\xi_{a_{i}: n}$.

Then, we can approximate the functions by the equations,

$$
\frac{f\left(z_{a_{i}: n}\right) z_{a_{i}: n}-f\left(z_{z_{i-1}: n}\right) z_{a_{i-1}: n}}{F\left(z_{a_{i}: n}\right)-F\left(z_{a_{i-1}: n}\right)} \simeq \alpha_{3 i}+\beta_{3 i} z_{a_{i}: n}+\gamma_{3 i} z_{a_{i-1}: n}
$$

where

$$
\begin{aligned}
& \alpha_{3 i}=\frac{q_{a_{i}: n} \xi_{a_{i}: n}^{2}-q_{a_{i-1}: n} \xi_{a_{i-1}: n}^{2}}{p_{a_{i}: n}-p_{a_{i-1}: n}}+\left[\frac{q_{a_{i}: n} \xi_{a_{i}: n}-q_{a_{i-1}: n} \xi_{a_{i-1}: n}}{p_{a_{i}: n}-p_{a_{i-1}: n}}\right]^{2}, \\
& \beta_{3 i}=\frac{q_{a_{i}: n}}{p_{a_{i}: n}-p_{a_{i-1}: n}}\left[1-\xi_{a_{i}: n}-\frac{q_{a_{i}: n} \xi_{a_{i}: n}-q_{a_{i-1}: n} \xi_{a_{i-1}: n}}{p_{a_{i}: n}-p_{a_{i-1}: n}}\right] \text {, } \\
& \gamma_{3 i}=-\frac{q_{a_{i-1}: n}}{p_{a_{i}: n}-p_{a_{i-1}: n}}\left[1-\xi_{a_{i-1}: n}-\frac{q_{a_{i}: n} \xi_{a_{i}: n}-q_{a_{i-1}: n} \xi_{a_{i-1}: n}}{p_{a_{i}: n}-p_{a_{i-1}: n}}\right] .
\end{aligned}
$$

By substituting (3.5) into (2.6), we may approximate the equations in (2.6) by

$$
\begin{aligned}
\frac{\partial \ln L}{\partial \sigma} \simeq & \frac{\partial \ln L^{* *}}{\partial \sigma}=-\frac{1}{\sigma}\left[u-\sum_{i=1}^{u} z_{a_{i}: n}-\left(n-a_{u}\right) z_{D}\right. \\
& \left.+\sum_{i=2}^{u} l_{i-1}\left(\alpha_{3 i}+\beta_{3 i} z_{a_{i}: n}+\gamma_{3 i} z_{a_{i-1}: n}\right)\right] \\
& =0 .
\end{aligned}
$$

We can derive AMLE as follows;

$$
\hat{\sigma}_{\mathrm{II}}=\frac{B_{\mathrm{II}}}{C_{\mathrm{II}}}
$$


where

$$
\begin{aligned}
& B_{\mathrm{II}}=\sum_{i=1}^{u} x_{a_{i}: n}+\left(n-a_{u}\right) D-\sum_{i=2}^{u} l_{i-1}\left(\beta_{3 i} x_{a_{i}: n}+\gamma_{3 i} x_{a_{i-1}: n}\right), \\
& C_{\mathrm{II}}=u+\sum_{i=2}^{u} l_{i-1} \alpha_{3 i} .
\end{aligned}
$$

To develop interval estimation for the $\sigma$, we need to compute the asymptotic variance which is related to the Fisher information. From (3.6), we obtain

$$
E\left(-\frac{\partial^{2} \ln L^{* *}}{\partial \sigma^{2}}\right)=\frac{D^{* *}}{\sigma^{2}}
$$

where

$$
\begin{aligned}
D^{* *}= & 2\left[\sum_{i=1}^{u} E\left(z_{a_{i}: n}\right)+\left(n-a_{u}\right) E\left(z_{D}\right)-\sum_{i=2}^{u} l_{i-1}\left(\beta_{3 i} E\left(z_{a_{i}: n}\right)+\gamma_{3 i} E\left(z_{a_{i-1}: n}\right)\right)\right] \\
& -\sum_{i=2}^{u} l_{i-1} \alpha_{3 i}-u .
\end{aligned}
$$

This quantity may be computed by using Balakrishnan and Cohen (1991):

$$
E\left(z_{i: n}\right)=\frac{n !}{(i-1) !(n-i) !} \sum_{m=0}^{i-1}(-1)^{m}\left(\begin{array}{c}
i-1 \\
m
\end{array}\right)(n+m-i+1)^{-2},
$$

The asymptotic variance of the $\mathrm{AMLE}_{\text {II }}$ of $\sigma$ is given by

$$
\operatorname{Var}\left(\hat{\sigma}_{\mathrm{II}}\right) \simeq \frac{1}{E\left[-\partial^{2} \ln L^{* *} / \partial \sigma^{2}\right]} .
$$

\section{Illustrative example and simulated results}

In this section, we present examples to illustrate the methods and assess the performance of estimators discussed in the previous sections.

\subsection{Real data}

Nelson (1982) gives data on times to breakdown of an insulating fluid in an accelerated test conducted at various test voltage. The data are as follows:

$\begin{array}{cccccccccc}0.19 & 0.78 & 0.96 & 1.31 & 2.78 & 3.16 & 4.15 & 4.67 & 4.85 & 6.50 \\ 7.35 & 8.01 & 8.27 & 12.06 & 31.75 & 32.52 & 33.91 & 36.71 & 72.89 & \end{array}$

For this data set, Balakrishnan and Sandu (1996) indicated that the one-parameter exponential distribution provides a satisfactory fit. In this example, we assume that the underlying distribution of this data is the exponential distribution based on the multiply Type I hybrid censoring scheme (i.e., $n=19, T=30, r=12$, and $a_{i}=1 \sim 2,4 \sim 19$ ). From (3.4), and (3.7), the AMLEs $\hat{\sigma}_{\mathrm{I}}=18.9059$, and $\hat{\sigma}_{\mathrm{II}}=18.5522$ are obtained. The MLE of $\sigma$ is numerically found in this case to be $\hat{\sigma}=18.3325$ 


\subsection{Simulation results}

To compare the performance of the proposed estimators of $\sigma$, we simulated the RMSE and bias of all proposed estimators, by employing the Monte Carlo simulation method. The multiply Type I hybrid censored samples for sample size $n=20$ and 40, and various censoring schemes from the standard exponential distribution are generated. Using this data, the RMSEs of all proposed estimators are calculated. We mainly compare the performances of the proposed estimators of $\sigma$, in terms of their biases and RMSEs for various censoring schemes. The simulation results are presented in Table 4.1, respectively.

From Table 4.1, the following general observations can be made. For all the methods, the RMSEs and biases of all estimators decrease as sample size $n$ increases. For fixed sample size $n$, the RMSE and bias increase generally as the pre-fixed number $r$ decreases. For fixed sample size $n$ and pre-fixed number $r$, the RMSE and bias decrease generally as the number of pre-fixed time $T$ increases. From Table 4.1, we observed that the MLE and $\hat{\sigma}_{\text {II }}$ are almost same values in terms of RMSE and bias. It is also seen that the RMSE and bias of the MLE and $\hat{\sigma}_{\text {II }}$ are generally more efficient than the $\hat{\sigma}_{\mathrm{I}}$.

Table 4.1 The RMSEs, and biases of the MLE, and AMLEs

\begin{tabular}{|c|c|c|c|c|c|c|}
\hline \multirow[b]{2}{*}{$n$} & \multirow[b]{2}{*}{$T$} & \multirow[b]{2}{*}{$a_{i}$} & \multirow[b]{2}{*}{$r$} & \multicolumn{3}{|c|}{ RMSE (Bias) } \\
\hline & & & & MLE & $\mathrm{AMLE}_{\mathrm{I}}$ & $\mathrm{AMLE}_{\mathrm{II}}$ \\
\hline \multirow{24}{*}{20} & \multirow{12}{*}{1.5} & \multirow{3}{*}{$1 \sim 4,6 \sim 20$} & 17 & $.28120(.03495)$ & $.29507(.05909)$ & $.28121(.03499)$ \\
\hline & & & 15 & $.28617(.02764)$ & $.29930(.05228)$ & $.28619(.02768)$ \\
\hline & & & 13 & $.28961(.01685)$ & $.30195(.04314)$ & $.28962(.01689)$ \\
\hline & & \multirow{3}{*}{$1 \sim 4,8 \sim 20$} & 17 & $.28079(.03663)$ & $.30111(.07053)$ & $.28089(.03680)$ \\
\hline & & & 15 & $.28225(.03542)$ & $.30236(.06934)$ & $.28235(.03563)$ \\
\hline & & & 13 & $.28719(.02810)$ & $.30603(.06274)$ & $.28727(.02832)$ \\
\hline & & \multirow{3}{*}{$1 \sim 2,8 \sim 20$} & 17 & $.28237(.03734)$ & $.30595(.07416)$ & $.28253(.03776)$ \\
\hline & & & 15 & $.28237(.03734)$ & $.30595(.07416)$ & $.25253(.03776)$ \\
\hline & & & 13 & $.28383(.03612)$ & $.30722(.07295)$ & $.28399(.03654)$ \\
\hline & & \multirow{3}{*}{$1 \sim 2,5 \sim 6,10 \sim 20$} & 17 & $.30798(.03938)$ & $.36195(.10777)$ & $.30815(.03977)$ \\
\hline & & & 15 & $.30798(.03938)$ & $.36195(.10777)$ & $.30815(.03977)$ \\
\hline & & & 13 & $.30932(.03817)$ & $.36293(.10662)$ & $.30949(.03856)$ \\
\hline & \multirow{12}{*}{1.8} & \multirow{3}{*}{$1 \sim 4,6 \sim 20$} & 17 & $.27024(.02814)$ & $.28173(.05101)$ & $.27026(.02818)$ \\
\hline & & & 15 & $.27471(.01680)$ & $.28528(.04052)$ & $.27472(.01684)$ \\
\hline & & & 13 & $.28024(.01112)$ & $.29104(.03694)$ & $.28025(.01116)$ \\
\hline & & \multirow{3}{*}{$1 \sim 4,8 \sim 20$} & 17 & $.26868(.03123)$ & $.28587(.06283)$ & $.26876(.03143)$ \\
\hline & & & 15 & $.27136(.02857)$ & $.28810(.06032)$ & $.27144(.02877)$ \\
\hline & & & 13 & $.27578(.01721)$ & $.29111(.05017)$ & $.27587(.01743)$ \\
\hline & & \multirow{3}{*}{$1 \sim 2,8 \sim 20$} & 17 & $.27033(.03185)$ & $.29109(.06653)$ & $.27048(.03225)$ \\
\hline & & & 15 & $.27033(.03185)$ & $.29109(.06653)$ & $.27048(.03225)$ \\
\hline & & & 13 & $.27302(.02917)$ & $.29342(.06402)$ & $.27316(.02958)$ \\
\hline & & \multirow{3}{*}{$1 \sim 2,5 \sim 6,10 \sim 20$} & 17 & $.26724(.03052)$ & $.30569(.09303)$ & $.26739(.03089)$ \\
\hline & & & 15 & $.26724(.03052)$ & $.30569(.09303)$ & $.26739(.03089)$ \\
\hline & & & 13 & $.26990(.02785)$ & $.30751(.09067)$ & $.27005(.02822)$ \\
\hline \multirow{24}{*}{40} & \multirow{12}{*}{1.5} & \multirow{3}{*}{$1 \sim 10,12 \sim 40$} & 35 & $.18233(.00887)$ & $.18952(.02261)$ & $.18234(.00887)$ \\
\hline & & & 30 & $.18674(.00295)$ & $.19325(.01700)$ & $.18647(.00296)$ \\
\hline & & & 28 & $.18797(.00286)$ & $.19401(.01174)$ & $.18797(.00286)$ \\
\hline & & \multirow{3}{*}{$1 \sim 10,14 \sim 40$} & 35 & $.18193(.00903)$ & $.19060(.02904)$ & $.18194(.00906)$ \\
\hline & & & 30 & $.18399(.00742)$ & $.19239(.02757)$ & $.18400(.00746)$ \\
\hline & & & 28 & $.18629(.00299)$ & $.19423(.02345)$ & $.18630(.00303)$ \\
\hline & & \multirow{3}{*}{$1 \sim 8,14 \sim 40$} & 35 & $.18216(.00912)$ & $.19101(.02934)$ & $.18218(.00919)$ \\
\hline & & & 30 & $.18279(.00872)$ & $.19158(.02897)$ & $.18281(.00879)$ \\
\hline & & & 28 & $.18419(.00753)$ & $.19281(.02788)$ & $.18421(.00760)$ \\
\hline & & & 35 & $.18188(.00905)$ & $.19884(.04635)$ & $.18189(.00910)$ \\
\hline & & $1 \sim 7,10 \sim 11,15 \sim 40$ & 30 & $.18251(.00866)$ & $19937(.04599)$ & $.18253(.00870)$ \\
\hline & & & 28 & $.18390(.00746)$ & $.20032(.04504)$ & $.18391(.00751)$ \\
\hline & & & 35 & $.17690(.00528)$ & $.18318(.01834)$ & $.17691(.00528)$ \\
\hline & & $1 \sim 10,12 \sim 40$ & 30 & $.18041(.00164)$ & $.18643(.01218)$ & $.18041(.00164)$ \\
\hline & & & 28 & $.18275(.00547)$ & $.18848(.00910)$ & $.18276(.00547)$ \\
\hline & & & 35 & $.17541(.00635)$ & $.18333(.02532)$ & $.17542(.00638)$ \\
\hline & & $1 \sim 10,14 \sim 40$ & 30 & $.17898(.00220)$ & $.18627(.02172)$ & $.17899(.00224)$ \\
\hline & 1.8 & & 28 & $.18031(.00159)$ & $.18750(.01851)$ & $.18032(.00156)$ \\
\hline & & & 35 & $.17544(.00648)$ & $.18361(.02565)$ & $.17546(.00655)$ \\
\hline & & $1 \sim 8,14 \sim 40$ & 30 & $.17828(.00399)$ & $.18604(.02329)$ & $.17829(.00406)$ \\
\hline & & & 28 & $.17907(.00229)$ & $.18670(.02191)$ & $.17908(.00236)$ \\
\hline & & & 35 & $.17525(.00643)$ & $.19016(.04138)$ & $.17526(.00648)$ \\
\hline & & $1 \sim 7,10 \sim 11,15 \sim 40$ & 30 & $.17810(.00394)$ & $.19239(.03915)$ & $.17811(.00399)$ \\
\hline & & & 28 & $.17891(.00224)$ & $.19280(.03811)$ & $.17892(.00229)$ \\
\hline
\end{tabular}




\section{Conclusions}

In this paper, we proposed the multiply Type I hybrid censoring. We also consider the estimation for the exponential distribution based on multiply Type I hybrid censored sample. We provide the MLE of the $\sigma$ by numerical method. We also provide approximate MLE of the $\sigma$, and it can be obtained explicitly. We compare the performance of the proposed estimators by Monte Carlo simulations, MLE and $\hat{\sigma}_{\text {II }}$ work quite well.

\section{References}

Asgharzadeh, A. (2009). Approximate MLE for the scaled generalized exponential distribution under progressive Type II censoring. Journal of the Korean Statistical Society, 38, 223-229.

Balakrishnan, N. and Cohen, A. C. (1991). Order statistics and inference: Estimation methods, Academic Press, Boston.

Balakrishnan, N. and Sandu, R. A. (1996). Best linear unbiased and maximum mlikelihood estimation for exponential distributions under general progressive Type II censored sample. Sanky A: The Indian Journal of Statistics, $\mathbf{5 8}, 1-9$.

Cho, Y. S., Lee, C. S. and Shin, H. J. (2013). Estimation for the generalized exponential distribution under progressive type I interval censoring. Journal of the Korean Data \& Information Science Society, 24, 1309-1317.

Epstein, B. (1954). Truncated life tests in the exponential case. Annals of Mathematical Statistics, 25, $555-564$.

Kang, J. H. and Lee, C. S. (2013). Estimations of the skew parameter in a skewed double power function distribution. Journal of the Korean Data 8 Information Science Society, 24, 901-909.

Kang, S. B. (2003). Approximate MLEs for exponential distribution under multiply Type II censoring. Journal of the Korean Data 63 Information Science Society, 14, 983-988.

Kang, S. B. and Cho, Y. S. (1998). MRE for exponential distirubion under general progressive Type II censored samples. Journal of the Korean Data 83 Information Science Society, 9, 71-76.

Kang, S. B. and Park, S. M. (2005). Estimation for the exponentiated exponential distribution based on multiply Type II censored samples. The Korean Communications in Statistics, 12, 643-652.

Lee, J. C. and Lee, C. S. (2012). An approximate maximum likelihood estimator in a weighted exponential distribution. Journal of the Korean Data \&S Information Science Society, 23, 219-225.

Lee, K. J., Park, C. K. and Cho, Y. S. (2012). Estimation of the exponential distribution based on multiply progressive Type II censored sample. The Korean Communications in Statistics, 19, 697-704.

Nelson, W. (1982). Applied life data analysis, John Wiley \& Sons, New York.

Shin, H. J., Lee, K. H. and Cho, Y. S. (2010). Parameter estimation for exponential distribution under progressive Type I interval censoring. Journal of the Korean Data 83 Information Science Society, 21, 927-934.

Singh, U. and Kumar, A. (2007). Bayesian estimation of the exponential parameter under a multiply Type II censoring scheme. Austrian Journal of Statistics, 36, 227-238. 